\title{
The intrinsic role and mechanism of tumor expressed-CD38 on lung adenocarcinoma progression
}

\author{
Long Gao ${ }^{1,2}$, Yuan $\mathrm{Liu}^{1,2}$, Xiaohong Du ${ }^{3}$, Sai Ma ${ }^{1,2}$, Minmin $\mathrm{Ge}^{1,2}$, Haijun Tang ${ }^{1,2}$, Chenfeng Han ${ }^{1,2}$, Xin Zhao ${ }^{1,2}$, Yanbin Liu ${ }^{1,2}$, \\ Yun Shao ${ }^{1,2}$, Zhao $\mathrm{Wu}^{1,2}$, Lianjun Zhang ${ }^{1,2}$, Fang Meng $\mathbb{D}^{1,2 \bowtie}$ and F. Xiao-Feng Qin $\mathbb{1}^{1,2 \bowtie}$
}

(c) The Author(s) 2021

It has been recently reported that CD38 expressed on tumor cells of multiple murine and human origins could be upregulated in response to PD-L1 antibody therapy, which led to dysfunction of tumor-infiltrating $\mathrm{CD} 8^{+} \mathrm{T}$ immune cells due to increasing the production of adenosine. However, the role of tumor expressed-CD38 on neoplastic formation and progression remains elusive. In the present study, we aimed to delineate the molecular and biochemical function of the tumor-associated CD38 in lung adenocarcinoma progression. Our clinical data showed that the upregulation of tumor-originated CD38 was correlated with poor survival of lung cancer patients. Using multiple in vitro assays we found that the enzymatic activity of tumor expressedCD38 facilitated lung cancer cell migration, proliferation, colony formation, and tumor development. Consistently, our in vivo results showed that inhibition of the enzymatic activity or antagonizing the enzymatic product of CD38 resulted in the similar inhibition of tumor proliferation and metastasis as CD38 gene knock-out or mutation. At biochemical level, we further identified that CADPR, the mainly hydrolytic product of CD38, was responsible for inducing the opening of TRPM2 iron channel leading to the influx of intracellular $\mathrm{Ca}^{2+}$ and then led to increasing levels of NRF2 while decreasing expression of KEAP1 in lung cancer cells. These findings suggested that malignant lung cancer cells were capable of using CADPR catalyzed by CD38 to facilitate tumor progression, and blocking the enzymatic activity of CD38 could be represented as an important strategy for preventing tumor progression.

Cell Death and Disease (2021)12:680; https://doi.org/10.1038/s41419-021-03968-2

\section{INTRODUCTION}

Studies have demonstrated that CD38 is one of the hallmarks of immune cells, which expressed on variety of immune cells such as $B$ cells, NK cells, T cells, and macrophages, and involved in regulating the differentiation, activation, and tolerance of immune cells $[1,2]$. And the FDA has approved two CD38 antibodies (Daratumumab and Isatuximab) for the treatment of multiple myeloma due to the promising preclinical and clinical activities of CD38 in hematopoietic system [3, 4]. More recently, Chen L uncovered a novel mechanism that tumor expressed CD38 was upregulated in tumor-bearing mice that responding to PD-L1 antibody, which led to immune tolerance and resistance. And it needs to be further addressed whether PD-L1 combined with CD38 antibody treatment could overcome PD-L1 resistance in preclinical or clinical settings [5]. Indeed, the clinical outcomes of lung cancer patients treated with combining PD-L1 and CD38 antibody are not satisfactory [6]. Thus, intrinsic mechanisms for the role of CD38 in the solid tumor warrant further exploration.

Researches have proved that the elevation of CD38 expression plays an inverse role in the regulation of nicotinamide adenine dinucleotide $\left(\mathrm{NAD}^{+}\right)$and maintenance of $\mathrm{NAD}^{+}$homeostasis is critical for maintaining the normal function of the immune cells, such as cytotoxic (CD8+) T cells and macrophages [7, 8]. CD38 possesses $\mathrm{NAD}^{+}$glycohydrolase activity and ADP ribosyl cyclase activity, both of which could convert $\mathrm{NAD}^{+}$to $\mathrm{CADPR}$ as well as hydrolase activity that converted CADPR to ADPR $[9,10]$. The formation of CADPR and ADPR catalyzed by CD38 from NAD ${ }^{+}$are dominated by a series of amino acid residues especially Cysteine 123 and Cysteine205 (for mouse) as well as glutamate146 and glutamate226 (for human) [11, 12]. Both ADPR and CADPR are important second messengers via ryanodine receptors (RyRs) to mediate the transmembrane transport of $\mathrm{Ca}^{2+}$ from endoplasmic reticulum in hematopoietic cells $[13,14]$. In addition, the level and enzymatic activity of CD38 gradually comes to increase during aging and leads to $\mathrm{NAD}^{+}$decline. Inhibiting the function of CD38 ameliorates age-related metabolic dysfunction [15].

But it is controversial about the role of tumor expressed CD38 and its enzymatic activity in the non-hematogenic tumor environment. A study had reported that CD38 ${ }^{\text {lo }}$ prostate cancer cells exhibited stem-like characteristics and surrounded with inflammatory cells in the glands, which inducing cancerous transformation of prostate cells and negatively correlated with prognosis [16]. In contrast, Bu X suggested that knocking out of CD38 in lung cancer cells resulted in an upregulation of $\mathrm{NAD}^{+}$,

\footnotetext{
${ }^{1}$ Institute of Systems Medicine, Chinese Academy of Medical Sciences and Peking Union Medical College, Beijing 100005, China. ${ }^{2}$ Suzhou Institute of Systems Medicine, Suzhou

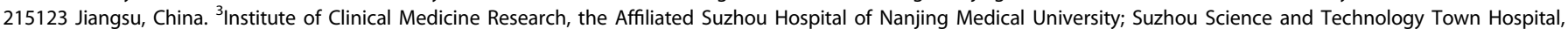
Suzhou, China. ${ }^{凶}$ email: onionmf@163.com; fqin1@foxmail.com
}

Edited by A. Peschiaroli 
A

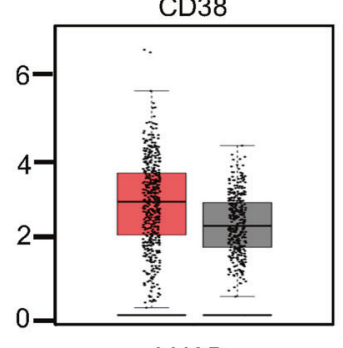

LUAD

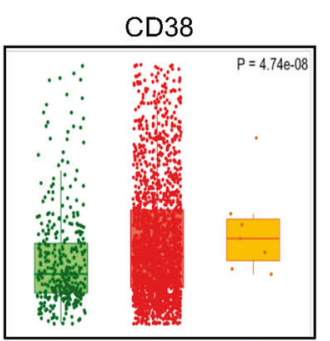

Normal Tumor Metastatic LUAD
B

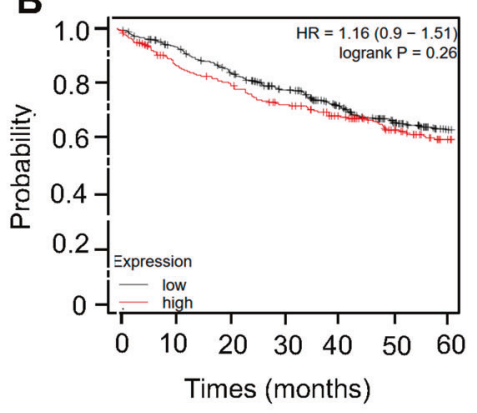

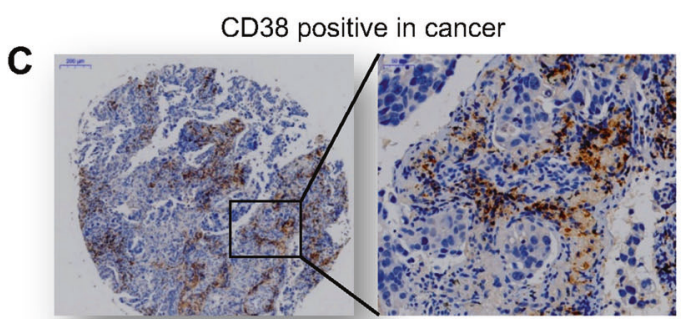

CD38 negative in cancer

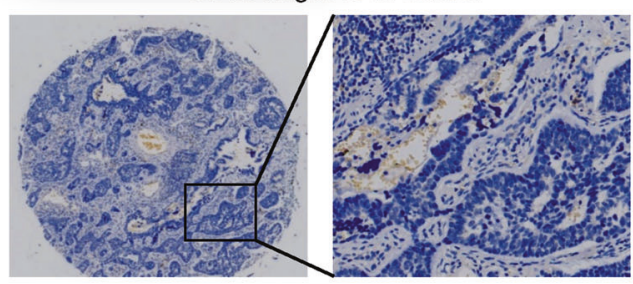

CD38 positive in adjacent

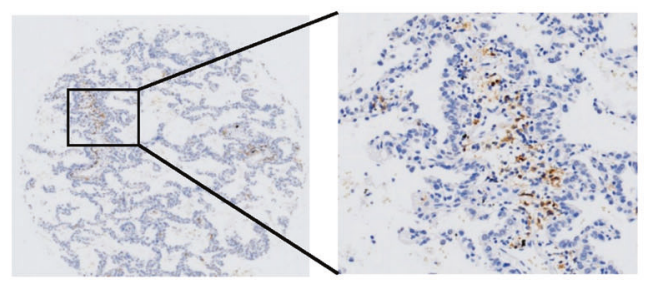

CD38 negative in adjacent

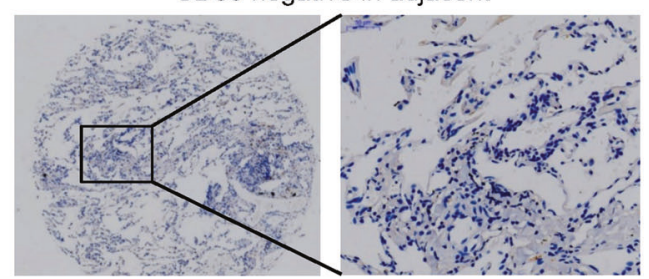

D

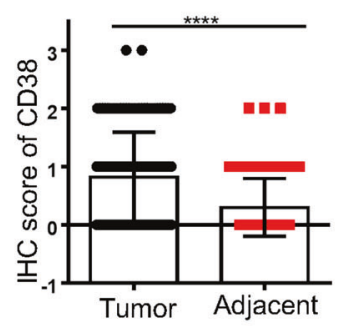

G

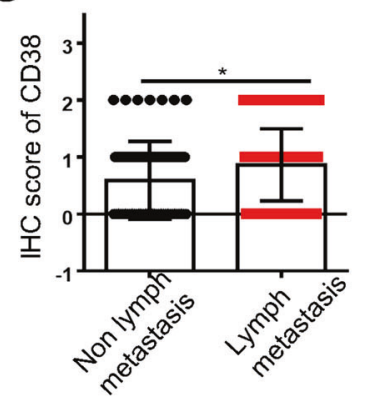

E

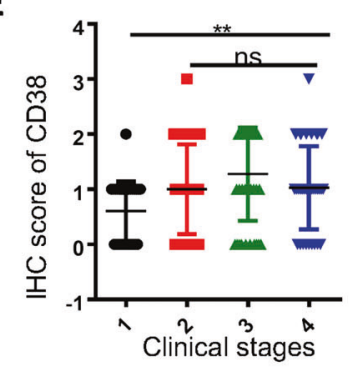

H

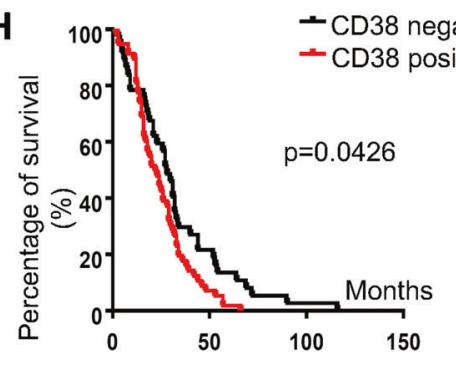

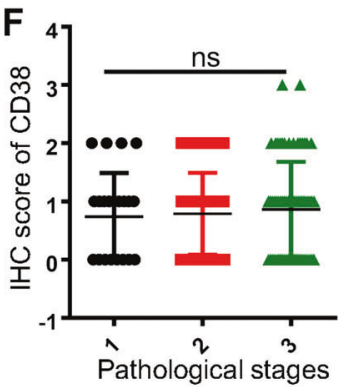

Pleural effusion

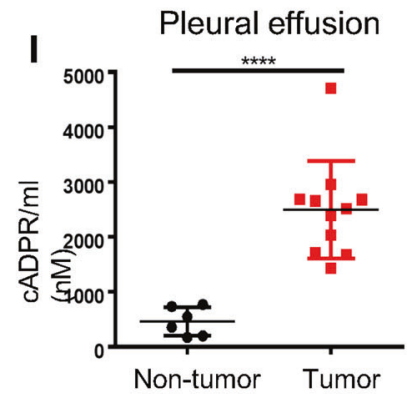

Fig. 1 The expression of CD38 in tumor microenvironment predicted a poor outcome. A Public data GEPIA and TNM plot database were used to analysis of the significant expression of CD38 in lung normal tissues, lung adenocarcinoma, and metastatic lung adenocarcinoma. B Kaplan-Meier Plotter database was selected to analysis the prognosis of CD38 in lung adenocarcinoma. C IHC staining for CD38 were performed from the lung adenocarcinoma and adjacent normal patient tissue microarray $(n=158)$. Representative images of cell staining intensity were shown. $\mathbf{D}$ Statistical analysis of the expression of CD38 in cancer specimens compared with adjacent normal was shown. E-G The percentage of CD38 positive cells were analyzed and used to generate an IHC-score for each sample that passed quality control. Statistical analysis of the relation of clinical stage (E), pathological stage $(\mathbf{F})$, lymph metastasis $(\mathbf{G})$ with CD38 expression in tumor specimens was shown. $\mathbf{H}$ Correlation of CD38 expression with the overall survival was performed by Kaplan-Meier OS analysis. I The pleural effusion from patients suffer with adenocarcinoma and non-tumorous control were detected by Amplite TM Fluorimetric cADP-Ribose Assay Kit to measure CADPR. $P$ values were calculated with ANOVA test. $P$ values were calculated with ANOVA test, ns, no significant difference; ${ }^{*} P<0.05 ;{ }^{* *} P<0.01 ;{ }^{* * *} P<0.001$. 
Table 1. Clinical characteristics of the 158 NSCLC patients.

\begin{tabular}{|c|c|c|c|c|}
\hline \multirow[t]{2}{*}{ Characteristics } & \multirow[t]{2}{*}{ Number } & \multicolumn{2}{|l|}{ Results } & \multirow[t]{2}{*}{$p$-value } \\
\hline & & Positive & Negative & \\
\hline Age (years) & 156 & & & 0.2002 \\
\hline$<60$ & 105 & 82 & 23 & \\
\hline$>60$ & 53 & 35 & 18 & \\
\hline Gender & 158 & & & 0.5075 \\
\hline Male & 78 & 53 & 27 & \\
\hline Female & 78 & 47 & 31 & \\
\hline Adenocarcinoma stage & 135 & & & 0.0947 \\
\hline Poorly & 41 & 23 & 18 & \\
\hline Moderately & 51 & 30 & 21 & \\
\hline advanced & 43 & 33 & 10 & \\
\hline TNM stage & 156 & & & $0.0059^{* *}$ \\
\hline 1 & 42 & 15 & 27 & \\
\hline II & 40 & 12 & 28 & \\
\hline III & 34 & 15 & 19 & \\
\hline IV & 40 & 26 & 14 & \\
\hline Distant metastasis & 149 & & & 1.001 \\
\hline Yes & 39 & 26 & 13 & \\
\hline No & 110 & 72 & 38 & \\
\hline Chemotherapy & 64 & & & 1.000 \\
\hline Yes & 16 & 9 & 7 & \\
\hline No & 48 & 26 & 22 & \\
\hline
\end{tabular}

which led to increasing CD8 $+\mathrm{T}$ cell infiltration and predicted a better prognosis [17]. Orazio Fortunato identified that CD133+CXCR4+ lung cancer stem cells were able to initiate distant metastasis and led to a resistance for chemotherapy. They also found that CD133+CXCR4+ cancer stem cells evaded immune surveillance via increasing expression of CD38 and CD73 [18]. Understanding the essential mechanisms of CD38 employed by tumor cells in tumor microenvironment represents an attractive therapeutic opportunity to selectively target tumor cells.

Herein, we aimed to investigate whether tumor expressed CD38 could affect the proliferation, progression, and metastasis in vitro and in vivo. The hypothesis of this study was that the enzymatic activities of CD38 was essential in mediating cell survival and metastasis in tumor microenvironment. Furthermore, we hoped to find that blockade enzymatic activity or competitive inhibiting cADPR could be efficacious to counter the tumor.

\section{MATERIALS AND METHODS}

\section{Cell culture and transfection}

A549, MDA-MB-231, PNAC-1, PanCO2, SW480, HCT116, 293T, BEL7402 and HepG2 cells were cultured in DMEM while LLC cells were maintained in RPMI-1640. The small molecule inhibitors used in this work were seen in additional Table 1. siRNAs targeting TRPM2 (Genepharma, China) was carried out according to the instructions (seen in additional Table 2). CD38 and TRPM2 sgRNA were colonized into Lenti-V2, pX458 plasmid and then transfect into cells according to the manufacturer's protocol. The Lentiviral-FG-EF/HTLV vector was used for constructing CD38-overexpression after packaged in 293T cells using FuGENE (Promega, E2311) transfection. CD38-cysteine205 and cysteine123 mutation were performed with QuickMutation ${ }^{\mathrm{TM}}$ gene mutagenesis kit (Beyotime, D0206).
Similar methods to get human CD38 mutation (glutamate146 and glutamate226 mutation).

\section{HPLC analysis}

HPLC was conducted on Agilent 1290 infinity liquid chromatograph Plus C18 column $(2.1 \times 50 \mathrm{~mm} 1.8 \mu \mathrm{m})$, Acchrom X-Amide column $(4.6 \times$ $250 \mathrm{~mm} 5 \mu \mathrm{m}$ ), eluting with a linear gradient of $0-80 \% \mathrm{CH}_{3} \mathrm{CN}$ in triethylammonium acetate buffer within $30 \mathrm{~min}$ at the rate of $1000 \mu \mathrm{L} /$ min. The concentration of each substance and the mixed standard was dissolved in pure water. The injection volume was $1 \mu \mathrm{L}$. Specific details will be provided if request.

\section{Animal studies}

LLC cells were either injected into subcutaneous of female C57BL/6 mice at $2 \times 10^{5} /$ mouse. Tumor growth was measured every two days (volume $=\left(\right.$ length $\times$ width $\left.^{2}\right) / 2$ ). And $5 \times 10^{6}$ of A549 cells were subcutaneously injected into the right oxter of $B A L B / C$ nude mice and tumor growth was monitored once per weeks. In addition, C57BL/6 mice injected with $2 \times 10^{5}$ of LLC cells and treated with PBS (Control), a single dose of 78 c (i.p., $15 \mathrm{mg} / \mathrm{kg}$, at day 7) or 8 -Br-cADPR (i.p., $500 \mu \mathrm{g} / \mathrm{kg}$, at day 7 , every two days). All groups would be sacrificed and the weight of tumor also be recorded.

\section{Intracellular $\mathrm{Ca}^{2+}$ measurements}

LLC and A549 tumor cells were digested and re-suspended in Hanks buffer containing $1 \%$ FBS with permeable $\mathrm{Ca}^{2+}$ indicator Fluo-3 AM (1 $\left.\mu \mathrm{M}, \mathrm{BIOSS}\right)$. The intracellular $\mathrm{Ca}^{2+}$ changes and average fluorescence intensity were measured by the flow cytometer and analyzed by FlowJo software.

\section{Flow cytometry assays}

For apoptosis assays, Annexin V and PI staining was performed according to the manufacturer's instructions. For tumor-infiltrating immunocytes, tumor tissues were prepared as single cell suspension and blocked with anti-mouse CD16/32 (BD), and then added concurrently with antibodies (seen in additional Table 4). Analysis was performed through FlowJo software and using single-color compensation as controls.

\section{cADPR measurements}

The detection of CADPR from cell lines, cancerous and non-cancerous pleural effusion was carried out according to the instructions of the Amplite $^{\mathrm{TM}}$ Fluorimetric cADP-Ribose Assay Kit (Cat.20305). In brief, cells in logarithmic phase were separated by " $40: 40: 20$ with $0.1 \mathrm{M}$ formic acid based approach". prepared and added CADPR standards and test samples $(50 \mu \mathrm{L})$, and incubated for $1 \mathrm{~h}$ after adding ADRPC working solution $(50 \mu \mathrm{L})$; then added $40 \mu \mathrm{L}$ Quest Fluor ${ }^{\mathrm{TM}}$ NAD Probe plus $40 \mu \mathrm{L}$ Assay Solution and continued incubation for another $20 \mathrm{~min}$. Finally added $30 \mu \mathrm{L}$ enhancer solution and incubated for another $20 \mathrm{~min}$. Monitor fluorescence intensity detected at $\mathrm{Ex} / \mathrm{Em}=420 / 480 \mathrm{~nm}$ by Synergy plate reader (BioTek). Specific details will be provided if request.

\section{Transwell assays}

A549 and LLC cells were digested and appended into the upper chamber in serum-free medium. 20\% FBS medium was added to the downer-chamber, and related small molecular inhibitors were also mixed if necessary. Cells in the supreme chambers were removed and the other side of the chambers were fixed with $4 \%$ paraformaldehyde and then stained with $0.1 \%$ crystal violet and photographed at bright field microscope.

\section{Colony formation assays}

Cells were seeded at the density of 500 cells/well into 24-well plate and cultured for seven days. Cells were fixed with $4 \%$ paraformaldehyde and stained with $0.1 \%$ crystal violet solution, followed by image capture at bright field microscope.

\section{RNA-sequences and Real-time quantitative PCR assays}

For RNA-sequences, total RNAs from A549 cell lines (CD38 WT and KO) were extracted using the TRIzol (Invitrogen), according to the supplier's recommendations. mRNA expression profiling was performed accordance with the protocol described by the Illumina manufacturer (GenePharma, Suzhou, China). Additional analyses were performed using Bioconductor 
A

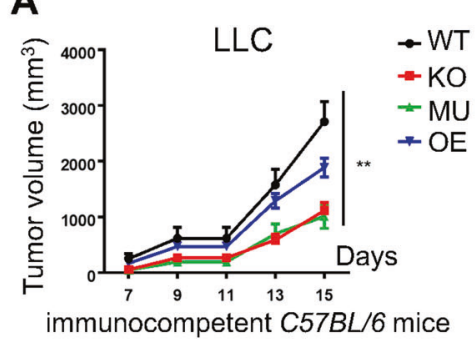

LLC

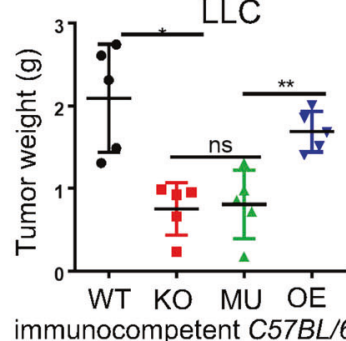

B

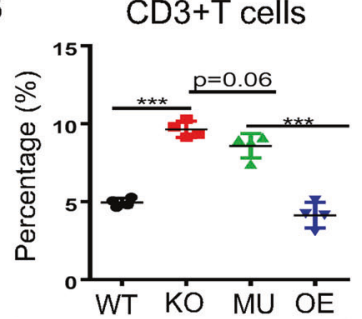

C

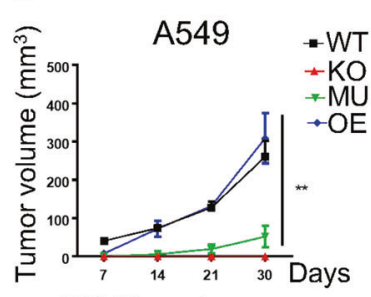

$B A L B / c$ nude mouse

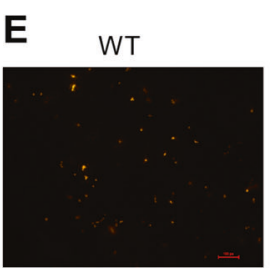

F

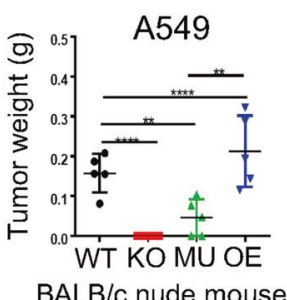

$B A L B / c$ nude mouse
D
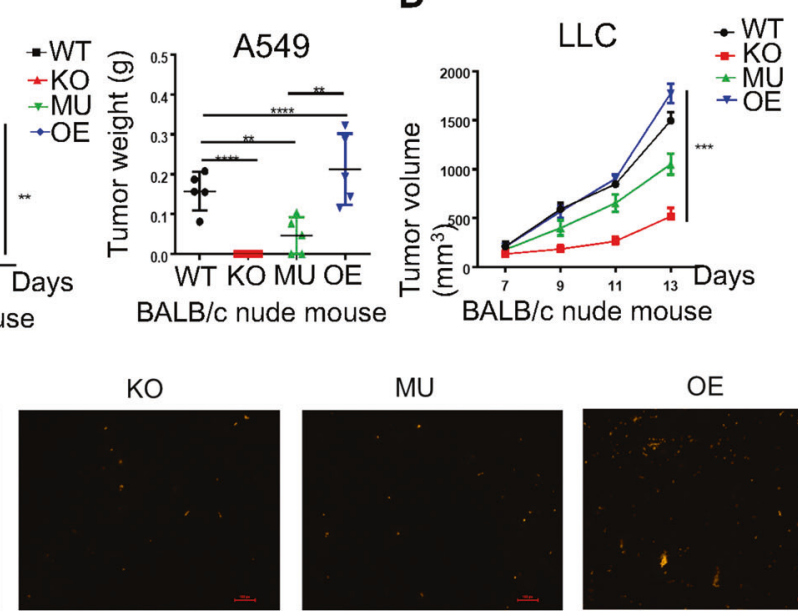

KO
MU

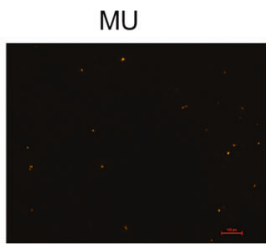

MU

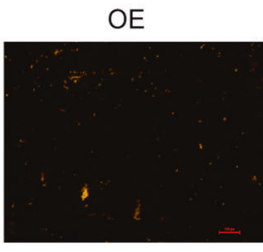

OE

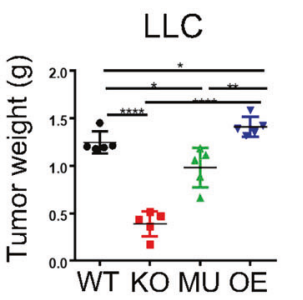

BALB/C nude mouse
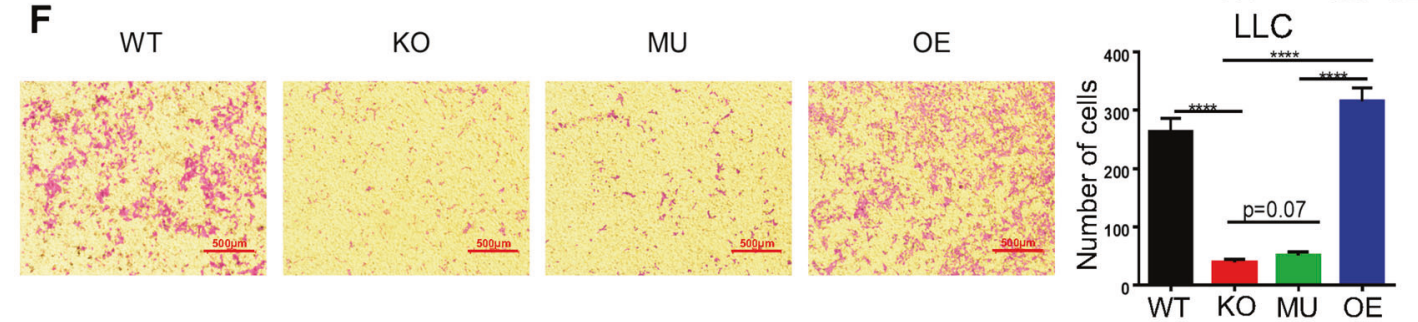

G

A549

LLC
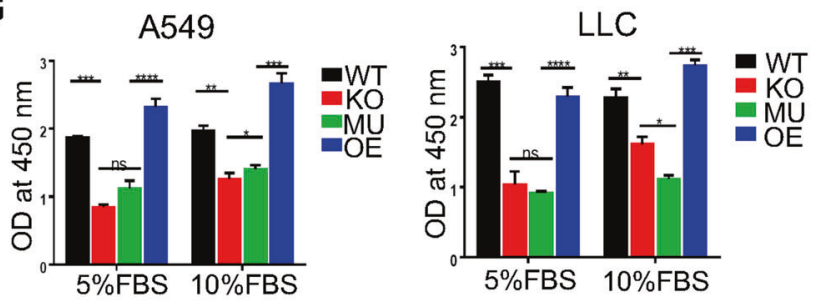

H

LLC
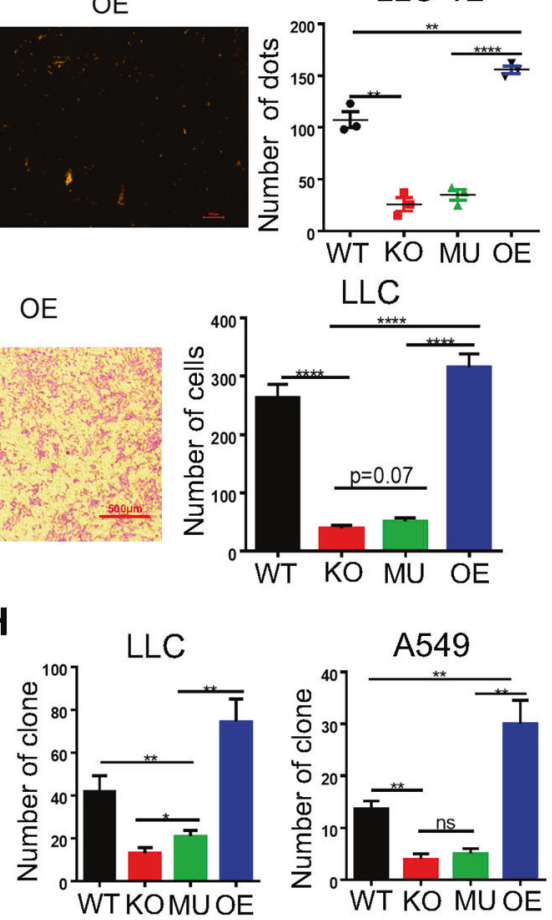

I
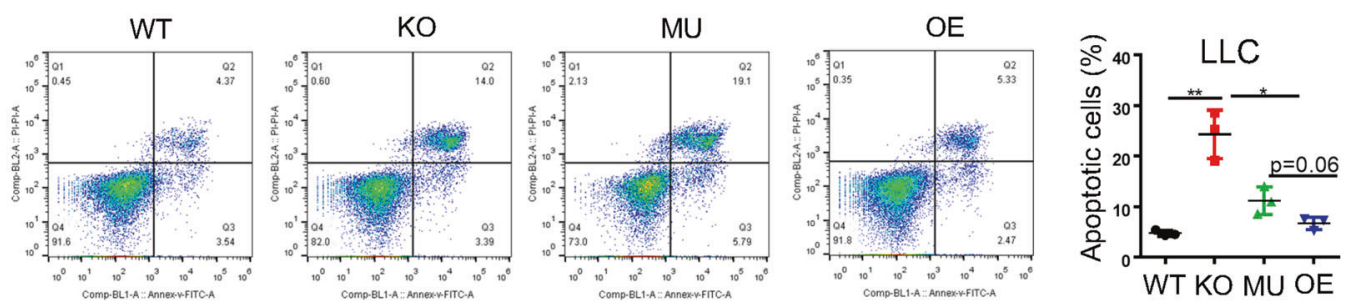

package Limma. For qRT-PCR, RNA were extracted by Trizol kit and reversed into CDNA solution. Quantitative PCR was designed by genespecific primers and GAPDH as reference gene (seen in additional Table 5). Samples were then analyzed for mRNA expression via qRT-PCR by using the LightCycler 480 (Roche) instrument and the relative expression of mRNA was normalized to the matched GAPDH.

\section{Western blotting assays}

Cells were lysed in RIPA lysis buffer and denatured in SDS loading buffer for total proteins. After denaturation, equal protein was separated by $10 \%$ SDS-PAGE and incubated with antibodies against CD38 (ThermoFisher, 1:500), KEAP-1 (Proteintech, 1:2000), NRF2 (CST, 1:2000), $\beta$-ACTIN (Proteintech,1:2500), GAPDH (Proteintech, 1:2000), TRPM2 (Abcam, 
Fig. 2 The enzymatic activity of CD38 was vital for cell proliferation, migration, and tumor progression. A LLC cells were separately implanted into immunocompetent C57BL/6 mouse (left). Tumors were measured every two days at day of 7. The tumor growth curve was shown with tumor sizes $(n=5)$. Mice were sacrificed at day of 15 after injection. The primary tumor mass is shown on the right (right). B FACS analysis showed the proportion of CD3 T+ tumor infiltrating lymphocyte (TIL) cells from primary tumors in immunocompetent mice $(n=5)$. C Growth of subcutaneous A549 tumors $\left(3 \times 10^{6}\right.$ cells/mouse) in immune-deficiency BALB/c-nude mice $(n=5)$ (left). Tumors were measured every week at beginning on day 7. Mice were sacrificed at day of 30 after injection. The primary tumor mass was shown on the right (right). D $1.2 \times 10^{5}$ of LLC tumor cells were implanted into BALB/c-nude mice $(n=5)$. The tumor growth curve was shown with tumor sizes (left). LLC tumorigenesis monitored every one day. Representative tumor mass was recorded after sacrificed at day of 13 after injection (right). E Number of spontaneous lung metastases in LLC-T2 tumor-bearing immunocompetent C57BL/6 mouse was shown. The visual numbers were randomly chosen in each group $(n=3)$, calculated by image J software. F LLC $\left(1.5 \times 10^{5}\right.$ cells per well) were seeded at the upper-chambers for $24 \mathrm{~h}$ and then the numbers of migrated cells that adhered to the lower surface of the trans-well chambers were counted under microscope randomly chosen visual fields per well within the area $(n=3)$. G LLC and A549 cells were seeded at a density of 3000 cells/well into $96-$ well plates for $48 \mathrm{~h}$ and then cell viability was determined using a CCK-8 assay, and the results were shown as the OD value to represent the relative proliferation ability of the cells $(n=3)$. H A549 cells were cultured at a density of 500 cells/well into 24 -well plates for 7 days and then the number of colony formation was visualized by staining with $0.1 \%$ crystal violet, and the results were shown as the number of positive area randomly $(n=3)$. I Representative FACS plots showing the apoptotic of LLC with different level of CD38 expression ( $n=3$ ). ANOVA or $t$ test was used to analyze the data. The data were presented as mean $\pm \mathrm{SEM}$, ns, no significant difference; ${ }^{*} P<0.05 ;{ }^{* *} P<0.01 ;{ }^{* * *} P<0.001$, $* * * * P<0.0001$

1:1500). Secondary antibodies were incubated for $1 \mathrm{~h}$ at room temperature, and visualized by the ECL substrate.

\section{Cell viability assays}

CCK-8 kits were used to estimate cell proliferation assays. Tumor cells were seeded in 96-well plates overnight then treated with different concentration of cADPR, 8-br-cAPDR, 2-APB, and ML-385 for $48 \mathrm{~h}$. And absorption at $450 \mathrm{~nm}$ was detected with a Synergy plate reader (BioTek).

\section{Immunohistochemistry assays and clinical data collection}

Tissues chips were purchased from Seville Biology Company (Wuhan, china) with $3 \mu \mathrm{m}$ sections. Chips underwent dewaxing, re-hydration, antigen retrieval, blocking, and then incubated with CD38 (ThermoFisher, 1:50) antibodies overnight. Sections were incubated with secondary antibody, washed and developed with DAB. Pictures were obtained using a Nikon microscope camera and NIS-Elements software and with a digital whole-slide scanner (Leica, SCN400F).

\section{Human clinical data analysis}

Genomic data of human specimens for subjects with Non-small-cell lung carcinoma were obtained from Kaplan-Meier Plotter (https://kmplot.com), the TNM plot database (https://www.tnmplot.com/) and GEPIA (http:// gepia.cancer-pku.cn). Kaplan-Meier survival analysis was conducted for evaluating the patient prognosis according to their correlation expression of CD38 and TRPM2 signature under alternative condition. Data were collected using empirical cumulative distribution function (ECDF) plots.

\section{Quantification and statistical analysis}

All data were presented as mean and error bars represent standard error of mean (SEM) from at least two or three biological replicates. Unpaired twotailed parametric $t$-test and Chi-square were calculated for statistical analyses. For survival analysis, the data were plotted and compared using the log-rank test. Pearson's correlation analysis was used to assess the correlation between genes. All statistical analysis was done using GraphPad Prism (v.6.0).

\section{RESULTS \\ The upregulation of CD38 was associated with tumor progression and predicted a poor outcome}

It remained unknown whether the expression of tumor CD38 was associated with the prognosis of lung adenocarcinoma patients. Here we employed tissue microarrays of lung adenocarcinoma specimens to analyze CD38 expression by immunohistochemically staining (Seville Biology Company (Wuhan, China), Chort No.1601). We identified that the expression of CD38 was related to a potentiality of metastasis but a shortened survival in lung adenocarcinoma (Fig. 1A, B). Among the 158 paired adjacent and cancer tissue microarrays, we found that $62 \%$ of cancer case exhibited positive staining for CD38, while only $28.3 \%$ were positive for adjacent tissue (Fig. 1C, D, $p<0.001$ ). The levels of CD38 in advanced tumors was significantly higher than that in early-stage (Fig. 1E, Table 1), but not associated with pathological characteristics (Fig. 1F). Our clinical data also demonstrated that CD38 was predicted a poor prognosis and promoted the metastasis (Fig. 1G, H). Then we collected a small scale of cancer-pleural effusion and non-cancerous-pleural effusion from volunteer patients, and found that the concentration of CADPR in cancerous pleural effusion was significantly higher than that of non-cancerous patients (Fig. 1I). We also confirmed that CD38 was mainly responsible for the production of CADPR in malignant pleural effusion, since no obvious CD157 was detected (Fig. S1). Taken together, these results indicated that CD38 revealed a poor survival of the lung adenocarcinoma patients.

\section{The enzymatic activity of CD38 was critical for lung cancer progression}

We initially confirmed that $\mathrm{CD} 38$ was highly expressed on the surface of A549 and LLC cells (Fig. S2A). To clarify the roles of CD38 in lung cancer, we constructed CD38 knocked out (KO) cells, CD38 mutant cells (A549-CD38 and LLC-CD38 mutant cell, MU) (Fig. S2A-C) $[11,12]$, and CD38 overexpressed cells (OE) (Fig. S2A-C). We also transfected a red fluorescent protein in LLC cells (LLC-T2), which would visualize tumor metastasis in vivo. No significant difference of tumor growth was observed between LLC-T2 and LLC in the mice model (Fig. S2D). We further illustrated that CD38 was certainly expressed on multiple tumor cell lines across human and murine species (Fig. S2E).

In immunocompetent mouse, we found that CD38 MU and KO tumor cells exhibited the impaired capability of tumor growth, mass and lung metastasis compared with CD38 WT and OE group (Fig. 2A, E). An increasing proportion of tumor-infiltrated $\mathrm{CD}^{+}$ $\mathrm{T}$ cells was observed in CD38 MU and $\mathrm{KO}$ group while macrophages or myeloid-derived suppressor cells (MDSCs) showed no differences among all the groups (Fig. 2B, Fig. S2F). Similar results were also obtained that growth of CD38 KO and MU cells was significantly reduced in size and mass compared with WT and $\mathrm{OE}$ in BALB/C-nude and CD38 deficiency mouse (Fig. 2C, D, Fig. S2G). In CD38 deficiency mouse, a rising number of $\mathrm{CD}^{+}$ $\mathrm{T}$ cells were also observed in CD38 MU and KO group (Fig. S2H). Consist with precious studies, we also demonstrated that tumor growth was dramatically inhibited in the CD38 deficiency mice in comparison with that of control WT mice (Fig. S2G, H).

In our vitro experiments, we observed that CD38 KO and MU cells exhibited an inhibition of cell proliferation, colony formation and migration but an increasing rate of apoptosis in both A549 and LLC lung cancer cells, and the overexpression of CD38 could rescue the ability of cell survival (Fig. 2F-I, Fig. S2I, J). Collectively, 
A
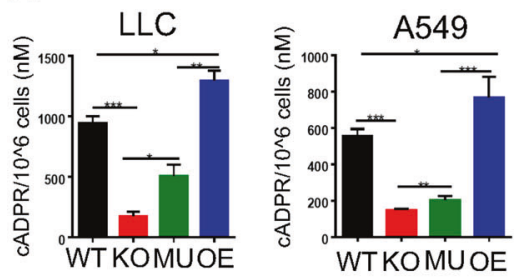

C WT

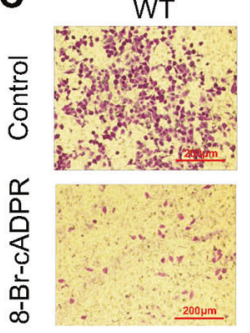

$\mathrm{KO}$

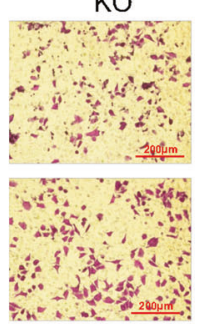

\section{B}

LLC
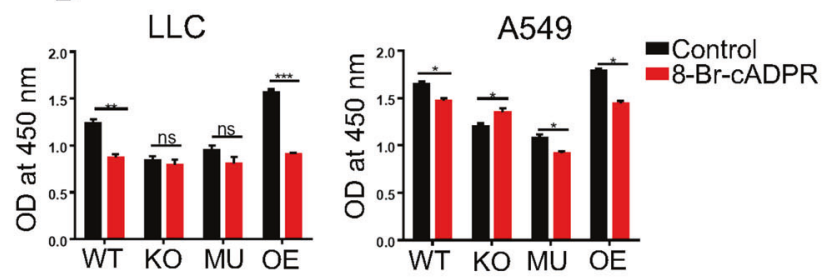

MU

OE
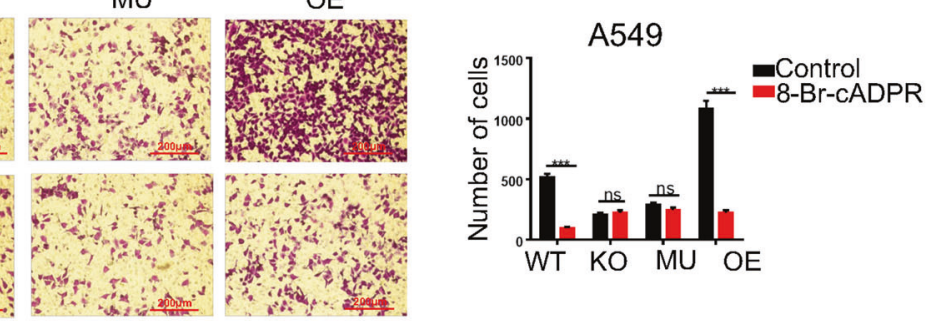

D
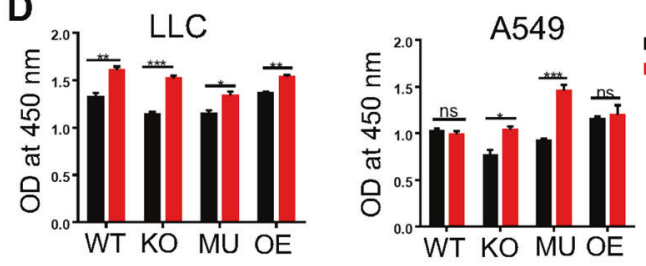

E

$\mathbf{F}$

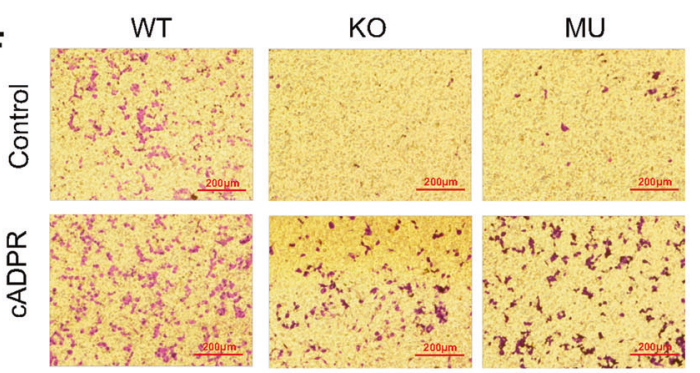

G

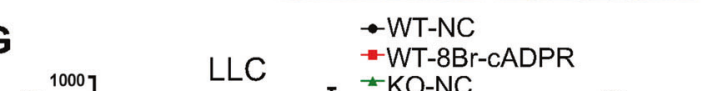
- Control
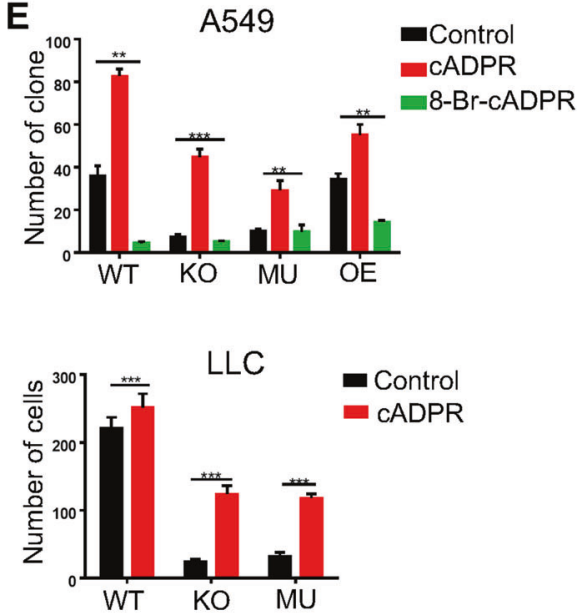

LLC
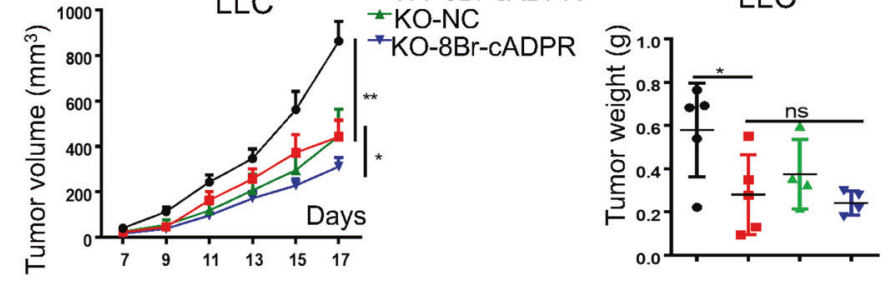

H

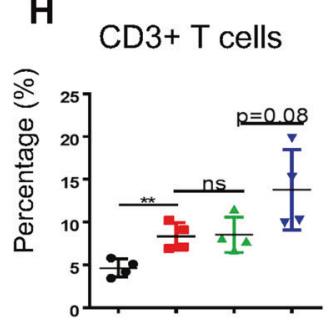

I
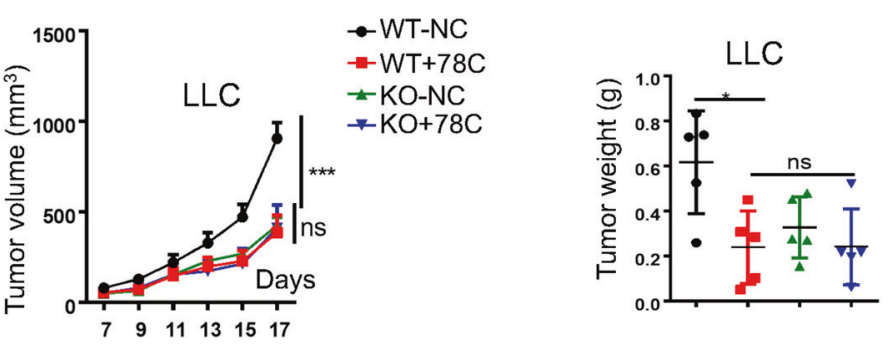

J CD3+ T cells

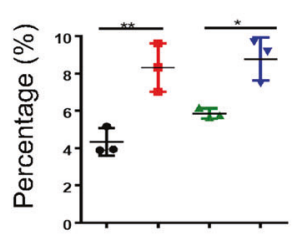

our data showed that the enzymatic activity of CD38 was required for tumor progression.

\section{CD38 utilized its hydrolytic product CADPR to promote tumor development}

As described previously, CD38 was well known as a major NAD glycohydrolase and participated in the generation of adenosine
(ADO) and our results showed that the enzymatic activity of CD38 was important for tumor progression. We thus investigated the changes of $\mathrm{NAD}^{+}$and adenosine (ADO) affected by CD38 enzymatic activity. Compared with CD38 WT cells, CD38 KO or MU cells led to an upregulation of $\mathrm{NAD}^{+}$but a declination of ADO (Fig. S3A). But we did not observe any significant changes of proliferation and migration in tumor cells after treated with $\mathrm{NAD}^{+}$ 
Fig. 3 CD38 used its hydrolyze product CADPR to promote tumor survival. A A549 and LLC tumor cells were subjected to Amplite ${ }^{T M}$ Fluorimetric CADP-Ribose Assay Kit to measure CADPR. The results were shown as the relative concentration of cADPR ( $n=3$ ). B LLC and A549 cells were seeded at a density 3000 cells/well into 96-well plates treated with 8-Br-cADPR (10 $\mu \mathrm{M}$ for LLC, $20 \mu \mathrm{M}$ for A549) for $48 \mathrm{~h}$ and then the cell viability was detected using a CCK-8 assay, and the results were represented as the OD value $(n=3)$. C A549 $\left(1.0 \times 10^{5} /\right.$ well) cells were seeded at the upper-chambers adding with 8-Br-cADPR ( $20 \mu \mathrm{M}$ for A549) for $24 \mathrm{~h}$ and then the numbers of migrated cells that adhered to the lower surface of the trans-well chambers were counted under an inverted microscope randomly chosen visual fields per well within the area $(n=3)$. D LLC and A549 cells were seeded at a density of 3000 cells/well into 96 -well plates administrated with cADPR (50 nM for LLC, $100 \mathrm{nM}$ for A549) for $48 \mathrm{~h}$ and then cell viability was detected using a CCK-8 assay, and the results were expressed as the OD value ( $n=3)$. E A549 cells were cultured at a density of 500 cells/well into 24-well plates treated with either 8-Br-cADPR (20 $\mu$ M for A549) or cADPR (100 nM for A549) for 7 days and then the number of colony formation was visualized by staining with $0.1 \%$ crystal violet, and the results were shown as the number of positive cells randomly $(n=3)$. F LLC $\left(1.5 \times 10^{5} /\right.$ well) cells were seeded at the upper-chambers adding with administrated with CADPR $(50 \mathrm{nM})$ for $24 \mathrm{~h}$ and then the numbers of migrated cells that adhered to the lower surface of the trans-well chambers were counted under an inverted microscope randomly chosen visual fields per well within the area $(n=3)$. G LLC cells were separately implanted into immunocompetent C57BL/6 mouse models $\left(2.0 \times 10^{5} / \mathrm{mouse}\right) .8-\mathrm{Br}$-cADPR ( $\left.500 \mu \mathrm{M} / \mathrm{kg}\right)$ or PBS was injected into mice (intraperitoneal) twice a week for 2 weeks beginning on day 5 after tumor cells implanted (left). Tumors were measured every two days at day of 7. Mice were sacrificed at day of 18 after injection. The tumor mass was shown with tumor sizes (right). H FACS analysis showed the proportion of CD3 T+ tumor infiltrating lymphocytes (TILs) cells from primary tumors in immunocompetent C57BL/6 mice $(n=5)$. I LLC cells were separately implanted into immunocompetent C57BL/6 mouse models $\left(2.0 \times 10^{5} / \mathrm{mouse}\right)$. $78 \mathrm{C}(10 \mathrm{mg} / \mathrm{kg})$ or a PBS control was injected into mice (intraperitoneal) twice a week for 2 weeks beginning on day 5 after LLC tumor cells were subcutaneously implanted (left). Tumors were measured every two days at day of 7 . Mice were sacrificed at day of 18 after injection. The tumor mass was shown with tumor sizes (right). J FACS analysis showed the proportion of CD3 T+ tumor infiltrating lymphocytes (TILs) cells from primary tumors in immunocompetent C57BL/ 6 mice $(n=5)$. Data were shown as mean \pm SEM. $P$ values were calculated with ANOVA or $t$ test. ns, no significant difference; ${ }^{*} P<0.05$; ${ }^{* *} P<0.01 ;{ }^{* * *} P<0.001,{ }^{* * *} P<0.001$.

A

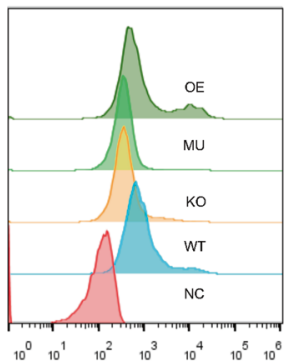

C

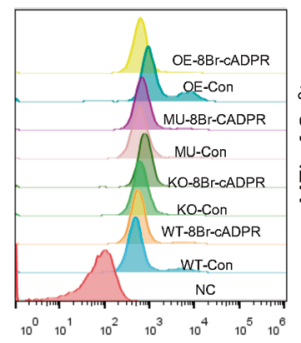

E

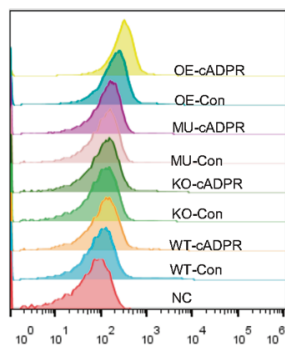

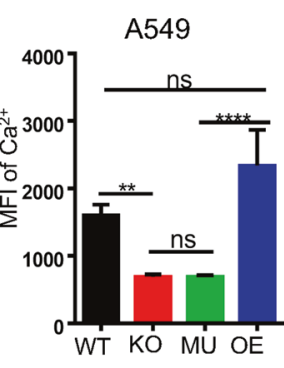

D
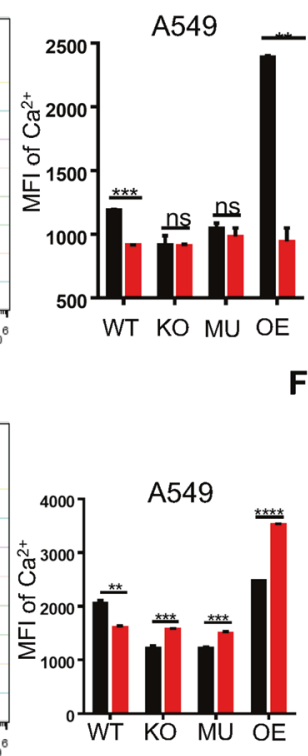

F
B
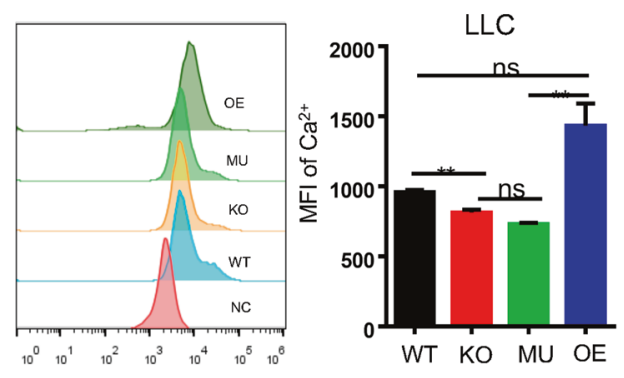

LLC
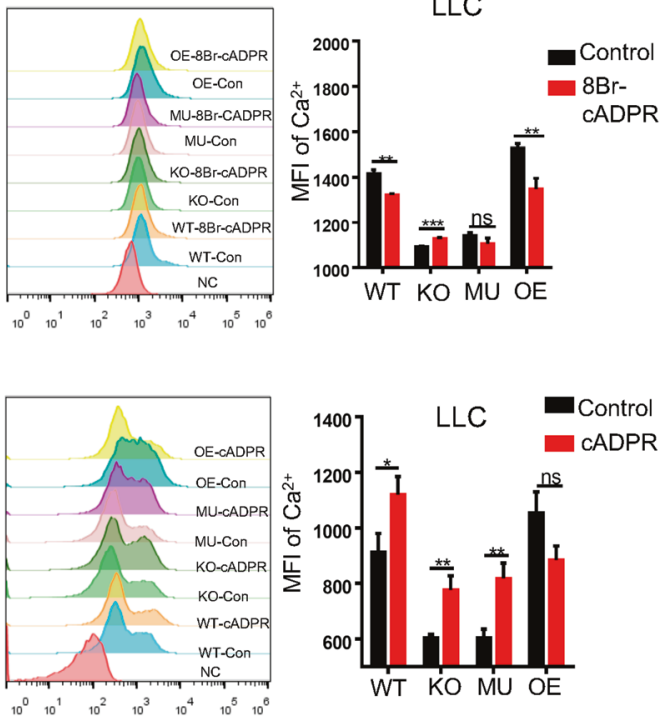

Fig. 4 CADPR promoted the concentration of cytoplasmic $\mathrm{Ca}^{2+}$. A, B FACS analysis was used to detect the MFI of cytoplasm Ca ${ }^{2+}$ in $\mathrm{A} 549(\mathbf{A}$, left) and LLC (B, right) tumor cells $(n=3)$. C, D FACS analysis showed the MFI of cytoplasm Ca ${ }^{2+}$ in A549 (C, left) and LLC (D, right) tumor cells treated with 8-Br-CADPR (for A549 was $20 \mu \mathrm{M}$, for LLC was $10 \mu \mathrm{M}$ ) for $1 \mathrm{~h}(n=3)$. E, F FACS analysis showed the MFI of cytoplasm Ca ${ }^{2+}$ in A549 (E, left) and LLC (F, right) tumor cells added with CADPR ((for A549 was $100 \mathrm{nM}$, for LLC was $50 \mathrm{nM})$ for $1 \mathrm{~h}$ ) for $0.5 \mathrm{~h}(n=3)$. Data were shown as mean \pm SEM. ANOVA or test was used to analyze data. ns, no significant difference; ${ }^{*} P<0.05 ;{ }^{*} P<0.01 ;{ }^{* *} P<0.001,{ }^{* * * *} P<0.0001$. 
A

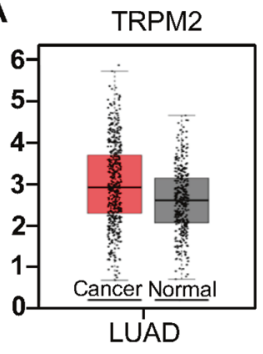

B

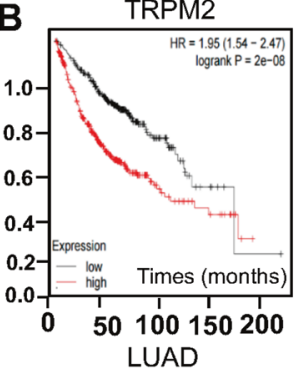

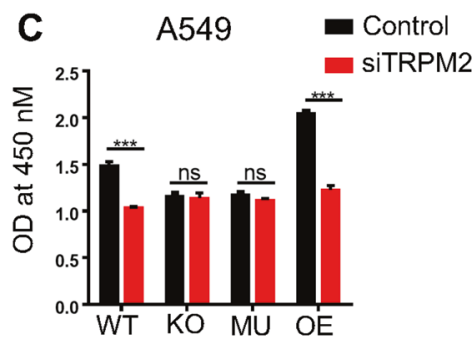

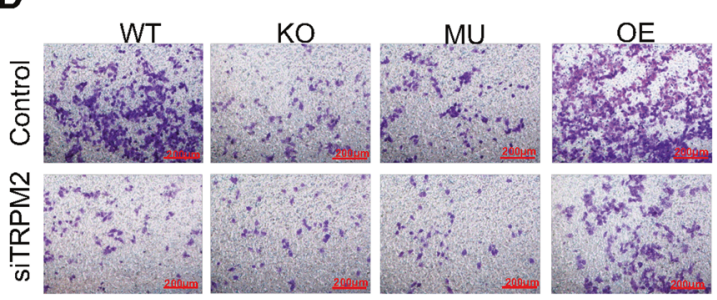

G

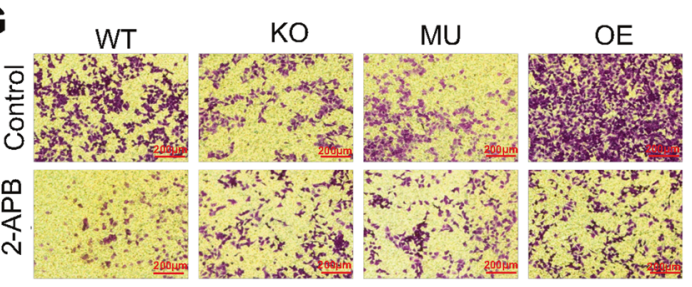

Control SiTRPM2 =
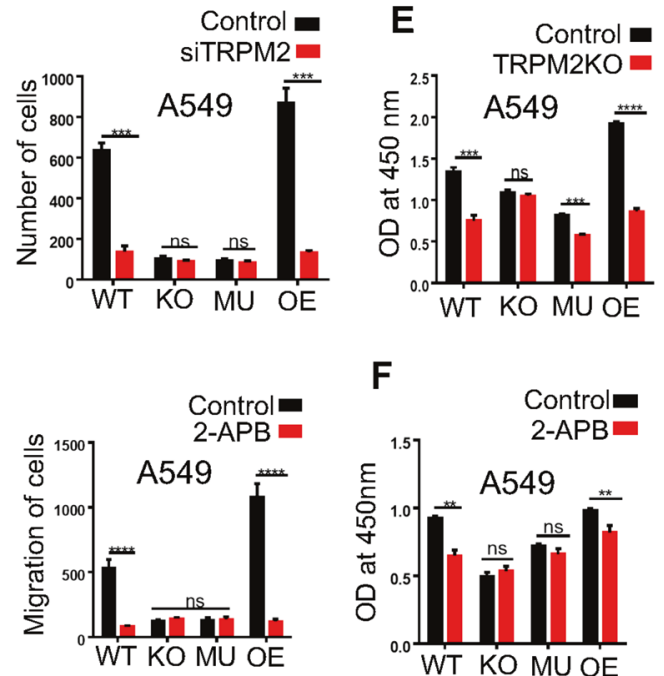

$\mathbf{F}$

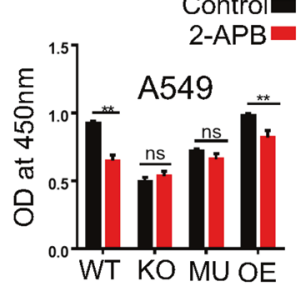

H
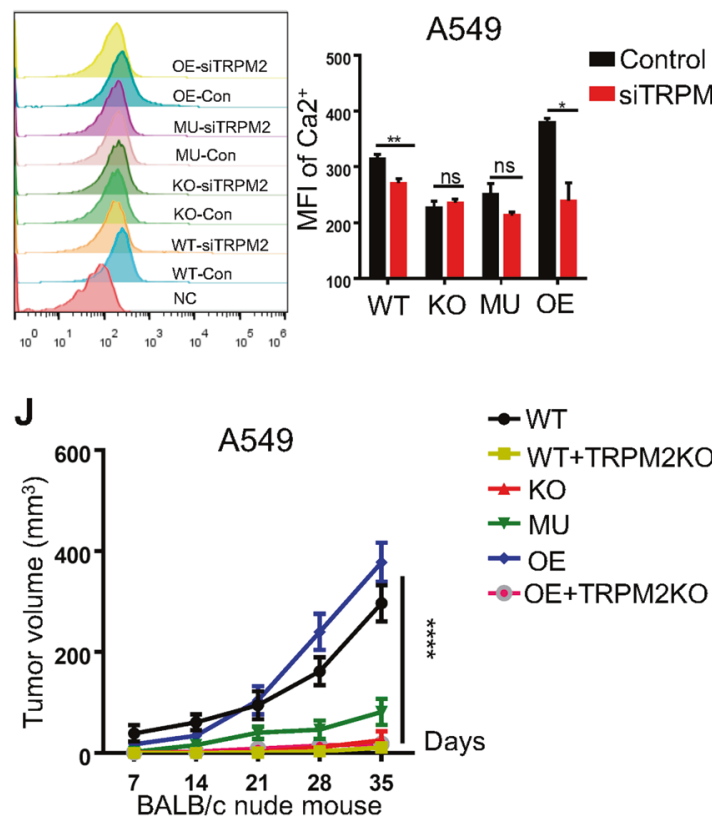

I

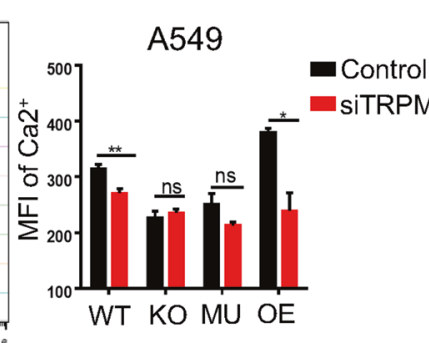

$10^{\circ}$

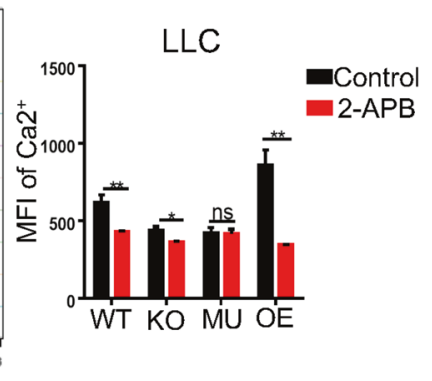

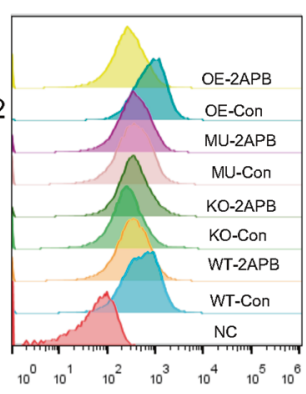

A549 (BALB/c nude mouse)

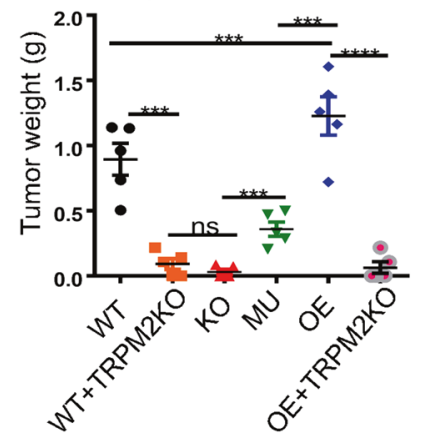

precursor NMN or ADO (Fig. S3B, C), indicated that CD38 might regulate tumor cell proliferation and migration independent on $\mathrm{ADO}$ and $\mathrm{NAD}^{+}$. We further showed that CD38 KO and MU cells displayed remarkably downregulated level of CADPR compared with WT and OE cells (Fig. 3A). And administration of 8-Br-CADPR (a competitive CD38 enzyme hydrolysate inhibitor) could result in a

significant inhibition of cell proliferation, colony formation, and migration on CD38 WT and OE tumor cells, but not on CD38 KO and MU cells (Fig. 3B, C, E, Fig. S3D). Furthermore, CADPR could rescue the cell proliferation, colony formation, and migration in the CD38 $\mathrm{KO}$ and MU tumor cells (Fig. 3D-F, Fig. S3E). These results suggested that CD38-CADPR signal was actually involved in regulation of cells 
Fig. 5 CADPR induced the concentration of intracellular $\mathbf{C a}^{2+}$ via TRPM2 channel. A GEPIA public database was used to assess the expression of TRPM2 in the lung adenocarcinoma compared with adjacent tissues. B Kaplan-Meier plotter database was used to perform the overall survival for lung adenocarcinoma tissue microarray with different TRPM2 levels. C A scramble control (TRPM2-con) or TRPM2 knock down (siTRPM2) A549 cells were seeded at a density of 3,000 cells/well into 96-well plates for $48 \mathrm{~h}$ and then cell viability was detected by using a CCK-8 assay, and the results were expressed as the OD value $(n=3)$. D A scramble control and TRPM2 knock down (siTRPM2) A549 (1 $\times 10^{5} /$ well) cells were seeded at the upper-chambers adding for $24 \mathrm{~h}$ and then the numbers of migrated cells that adhered to the lower surface of the trans-well chambers were counted under an inverted microscope randomly chosen visual fields per well within the area $(n=3)$. E TRPM2control or TRPM2-KO A549 tumor cells were seeded at a density of 3000 cells/well into 96 -well plates for $48 \mathrm{~h}$ and then cell viability was detected using a CCK-8 assay, and the results were expressed as the OD value $(n=3)$. F Control (TRPM2-con) or small molecular inhibitor (2APB, $200 \mu \mathrm{M})$ A549 cells were seeded at a density of 3000 cells/well into $96-$ well plates for $48 \mathrm{~h}$ and then cell viability was detected using a CCK-8 assay, and the results were expressed as the OD value $(n=3)$. G Control or a small molecular inhibitor $(2-\mathrm{APB}, 200 \mu \mathrm{M}) \mathrm{A} 549$ cells $(1 \times$ $10^{5} /$ well) were seeded at the upper-chambers adding for $24 \mathrm{~h}$ and then the numbers of migrated cells that adhered to the lower surface of the trans-well chambers were counted under an inverted microscope randomly chosen visual fields per well within the area $(n=3)$. $\mathbf{H}$ FACS analysis showed the MFI of cytoplasm $\mathrm{Ca}^{2+}$ in A549 cells with a scramble control (TRPM2-con) or TRPM2 knock down (siTRPM2) $(n=3)$. I FACS analysis was used to perform the MFI of cytoplasmic $\mathrm{Ca}^{2+}$ in LLC cells with control or a small molecular inhibitor $(2-\mathrm{APB}, 100 \mu \mathrm{M}, 1 \mathrm{~h})(n=3)$. J Growth of subcutaneous A549 tumors $\left(3 \times 10^{6}\right.$ cells per mouse) in immune-deficiency BALB/c-nude mice was performed $(n=5)$ (left). Tumors were measured every 2 days beginning on day 7 after injection. Mice were sacrificed at day of 35 after injection. The primary tumor mass was also shown on the right (right). Data were presented as mean \pm SEM. ANOVA or test was used to analyze data. ns, no significant difference; ${ }^{*} P<0.05$; ${ }^{* *} P<0.01$; ${ }^{* *} P<0.001$, ${ }^{* * * *} P<0.001$.

progression. We then examined the therapeutic effects of 8-BrCADPR in LLC-bearing immunocompetent mice. It was found that both 8-Br-cADPR and 78C (an enzyme inhibitor of CD38) could significantly inhibit tumor growth and tumor mass in CD38 WT-LLCtumor-bearing mice while no therapeutic effects were observed in CD38 KO mice (Fig. 3G). Similarly, we also found that 8-Br-cADPR and $78 \mathrm{C}$ could increase the infiltration of CD3+ T cells (Fig. $3 \mathrm{H}-\mathrm{J}$, Fig. S3F, G). In summary, our results demonstrated that CD38 mediated tumor cell progression through CD38-CADPR signaling and blockade this axis might potentially serve as a novel therapeutic intervention for lung cancer.

CADPR could promote the concentration of cytoplasmic $\mathrm{Ca}^{2+}$ It was reported that CADPR could modulate intracellular calcium in immune cells via the activation of RyRs anchored on the endoplasmic reticulum. $\mathrm{Ca}^{2+}$ is a dynamic second messenger induced by many factors, leading to pleiotropic effects on cell survival including changes of migration, proliferation, and metabolism. Thus, it is not surprising that aberrant regulation of $\mathrm{Ca}^{2+}$ signals can lead to pathological phenotypes, including cancer progression [19]. However, given the highly contextspecific nature of $\mathrm{Ca}^{2+}$ dependent changes in tumor cell function, its role in the TME needs to be further explored. Here we confirmed that CD38 KO and MU cells exhibited a lower concentration of cytoplasmic calcium than that of WT cells, while an increasing level of calcium were shown in CD38 OE cells (Fig. 4A, B). Exogenously administration of 8-Br-cADPR was found to induce a significantly decrease of the cytoplasmic calcium in CD38 WT and OE tumor cells but not in CD38 KO and MU cells (Fig. 4C, D). When stimulated with a minimal level of CADPR, the cytoplasmic calcium of CD38 KO and MU cells was rescued, and CADPR could further upregulate the level of calcium in WT and OE cells (Fig. 4E, F). Herein, our results demonstrated that CD38 might use CADPR to influence cytoplasmic calcium concentration of tumor cells.

\section{CADPR induced the concentration of intracellular $\mathrm{Ca}^{2+}$ via TRPM2 channel}

We then hypothesized that CADPR might regulate the influx of extracellular calcium by activating TRPM2, among which its NUDT9H domain could be activated by cADPR [20]. Using GEPIA database, we found that the expression of TRPM2 in tumor was higher than that of normal tissues (Fig. 5A) and predicted a poor prognosis of lung adenocarcinoma patients (Fig. 5B). Next, it was confirmed that TRPM2 was expressed both in A549 and LLC cells (Fig. S4A). Along with the down-regulation of TRPM2 by siRNA in A549 cells (Fig. S4B), the inhibition of cell proliferation and migration was mainly occurred in CD38 WT and OE cells (Fig. 5C, D). When further knocking out TRPM2 in A549 tumor cells (Fig. S4C), similar results were also observed (Fig. 5E). Furthermore, 2-APB exhibited similar affection on CD38 WT and OE cells (Fig. 5F, $\mathrm{G}$, Fig. S4D). This phenomenon might be explained by which the loss of CD38 enzyme activity resulted in the lack of CADPR and the inactivation of TRPM2 channel.

Along with inhibition of TRPM2 by siRNA or 2-APB, the decreasing concentration of calcium was observed in CD38 WT and $\mathrm{OE}$ tumor cell than that of control (Fig. $5 \mathrm{H}, \mathrm{I}$ ). Our results showed that all of TRPM2-deficient A549 tumor cells exhibited a significant reduction of tumor burden compared with that of control but independent on the expression of CD38 (Fig. 5J). Thus, we speculated that CD38-CADPR signal at least in partly through the activation of TRPM2 to promote the tumor survival. Further confirming the expression of TRPM 2 in a variety of human cell lines (Fig. S4E), we found that CADPR could promote cell proliferation and migration, while 8-Br-CADPR shown on the contrary (Fig. S4F, G). Altogether, our results strongly suggested that the generation of CADPR by CD38 via TRPM2 channel to promote the cell progression.

\section{CADPR regulated KEAP1/NRF2 signal pathway to facilitate tumor progression}

Analysis differences of pathways between A549 CD38 WT and CD38 KO tumor cells by Gene Ontology (GO) and Kyoto Encyclopedia of Genes and Genomes (KEGG), we identified cell cycle, cell proliferation, stress-activated MAPK signaling, cell metabolic, and other signaling pathways were changed (Fig. 6A, $B)$. And in-depth analysis of differentially expressed genes by realtime PCR, we obtained that the mRNA expression of Nrf2-target downstream anti-oxidative genes GCLC, AKP1B10, GSTM3, and NQO1 were significantly upregulated (Fig. 6B). The KEAP1-NRF2 pathway played vital roles in regulation of cancer progression and recent studies indicated that dysfunction of KEAP1 was cooperated to drive LUAD initiation and correlated with poor survival in patients [21-24]. So we hypothesized that CADPR might via KEAP1-NRF2 signal to regulate tumor progression.

CD38 KO and MU tumor cells showed a higher expression of KEAP1 than that of WT and OE cells, while NRF2 was on the contrary (Fig. 6C). By adding CADPR and 8-Br-CADPR to co-culture tumor cells, we found that CADPR inhibited the expression of KEAP1, but promoted the expression of NRF2 in CD38 KO and MU tumor cells, while 8-Br-cADPR was on the contrary (Fig. 6D). We also verified that the changes of KEAP1 and NRF2 were corresponded with TRPM2 KO in tumor cells (Fig. 6E). In addition, our results revealed that the capability of cell proliferation was 
A

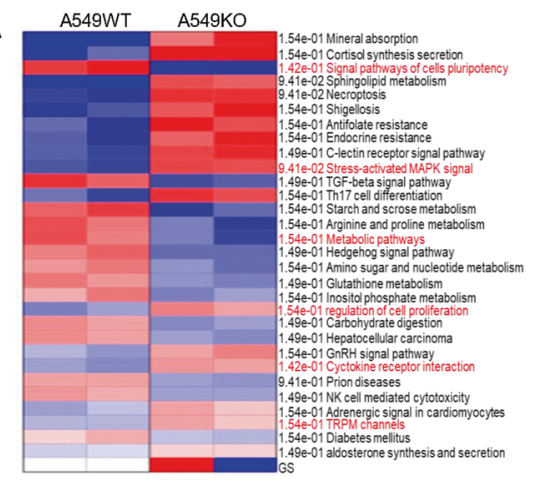

C

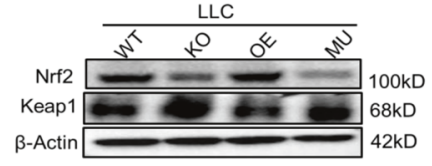

B
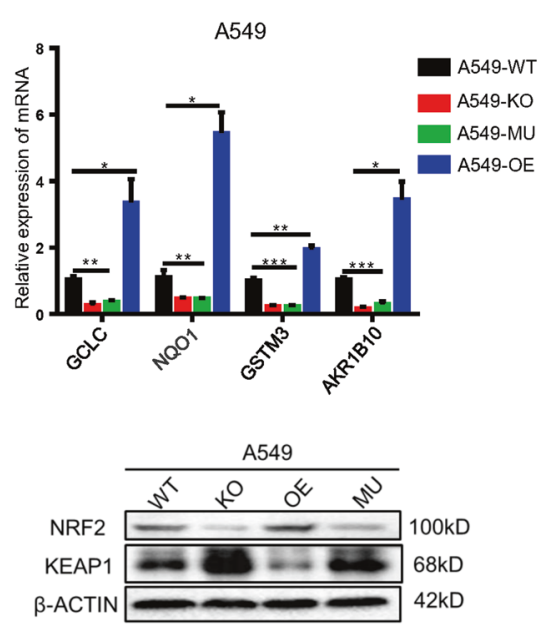
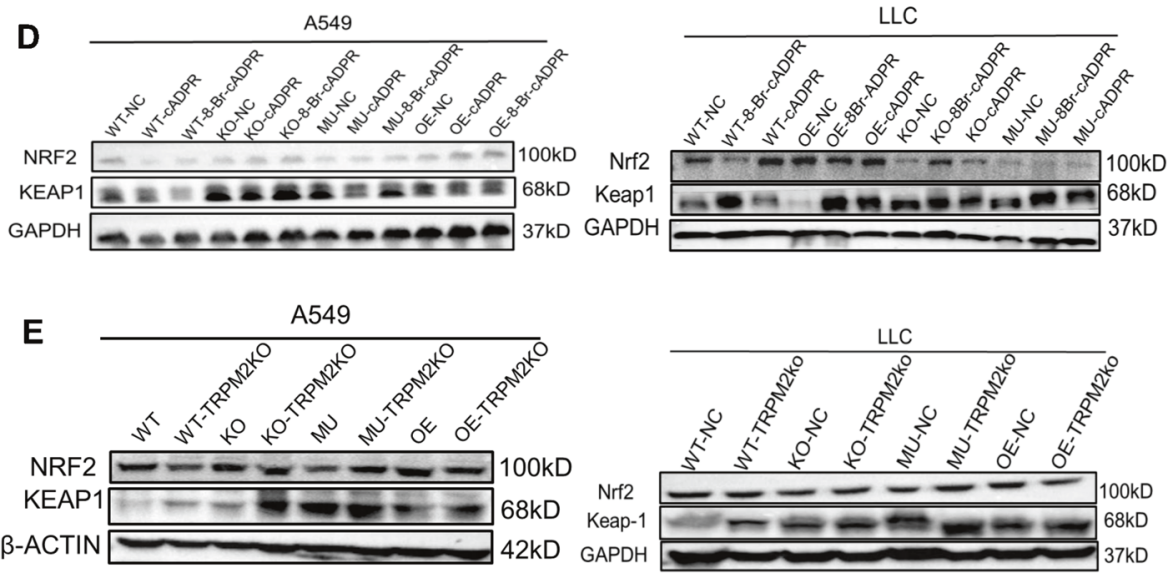

$\mathbf{F}$

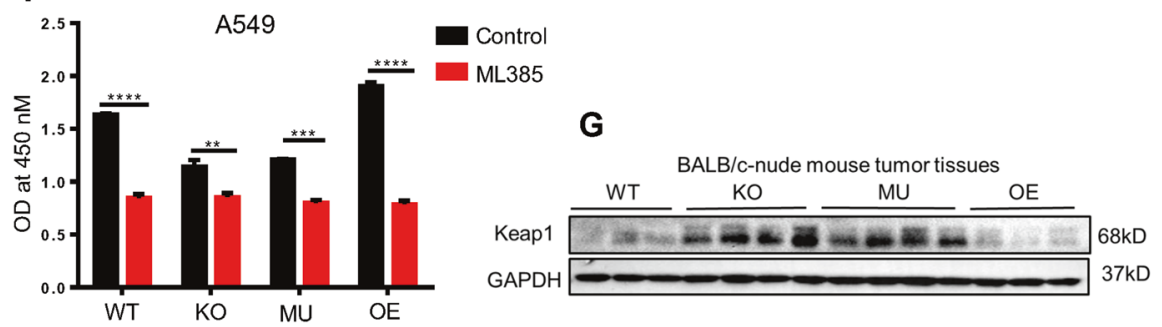

Fig. 6 CADPR regulated KEAP1/NRF2 signal pathway to facilitate tumor progression. A Heat map showed the significant differences of signaling pathways from A549 CD38 WT and CD38 KO tumor cells by Kyoto Encyclopedia of Genes and Genomes (KEGG) analysis. B Relative mRNA expression levels of GCLC, NQO1, GSTM3, and ARK1B10 were shown in A549 cells calculated by qRT-PCR. And GAPDH was used as internal control $(n=3)$. C Western blotting was confirmed the expression of KEAP1 and NRF2 in A549 and LLC cells. GAPDH was shown as control. D Western blotting was used to detect the expression of KEAP1 and NRF2 in A549 and LLC cells treated with control, cADPR (for LLC was $50 \mathrm{nM}$, for A549 was $100 \mathrm{nM}, 24 \mathrm{~h}$ ) and 8-Br-CADPR (for LLC was $10 \mu \mathrm{M}$, for A549 was $20 \mu \mathrm{M}, 24 \mathrm{~h}$ ). E Western blotting was used to analysis of the expression of KEAP1 and NRF2 in A549 and LLC cells treated with an empty control PX458-plasmid (TRPM2-NC) or PX458-sgTRPM2plasmid (sgTRPM2, TRPM2 KO). F A549 cells were seeded at a density of 3000 cells/well into 96 -well plates treated with ML385 (10 $\mu$ M) for $48 \mathrm{~h}$ and then cell viability was detected by using a CCK-8 assay, and the results were expressed as the OD value $(n=3)$. G Western blotting was used to analysis of the expression of Keap1 from LLC bearing-BALB/c-nude tumor tissues. Data were presented as mean \pm SEM. ANOVA or test was used to analyze data. ns, no significant difference; ${ }^{*} P<0.05 ;{ }^{* *} P<0.01 ;{ }^{* * *} P<0.001,{ }^{* * * *} P<0.001$.

notably restrained in all groups but CD38 WT and OE tumor cells showed a more significant inhibition than CD38 KO and MU tumor cells after treated tumor cells with ML385 (Fig. 6F). And in CD38 LLC-bearing-BALB/c-nude mice tumor tissues, it was also confirmed that CD38 KO and MU-bearing-tumor tissues exhibited a higher expression of Keap1 than that of in CD38 WT and OEbearing-tumor tissues (Fig. 6G). Altogether, these results indicated that KEAP1-NRF2 signal pathway was affected by CD38-CADPR signal.

\section{DISCUSSION}

Previous study clarified that PD-L1 combined with CD38 antibody could overcome PD-L1 resistance [5]. Herein, we provided a 
significant mechanism that the enzymatic activity of tumor expressed CD38 induced the opening of TRPM2 by generating CADPR, and resulted in an elevated concentration of cytoplasm calcium and facilitated primary tumor progression and metastasis by triggering KEAP1-NRF2 signal pathway. We also found targeting enzymatic activity of CD38 and its enzymatic product CADPR might be a promising strategy for the cancer therapy.

Indeed, in multiple myeloma, acute lymphoblastic leukemia, and other hematological cells, CD38 was abnormally upregulated and resulted in clinical manifestations of the disease include signs of organ dysfunction, along with an immunosuppressive microenvironment that finally led to hematological cells aggressiveness and poor prognosis [25, 26]. Various preclinical studies and ongoing clinical trials aimed to evaluate the efficiency of CD38 antibodies in hematological malignancies such as $\mathrm{NK} / \mathrm{T}$ cell lymphoma and acute lymphoblastic leukemia [27, 28]. An updated study uncovered a phenomenon that CD38-positive macrophages used extracellular enzyme activity to inhibit the $\mathrm{NAD}^{+}$levels of CD38-negative cells [8]. To date, there were no data regarding the role of CD38 in solid tumor environment. This question prompted us to investigate more in detail how the biological function of CD38 was involved in the solid tumor development, including (1) roles of CD38 ecto-enzymatic activity during tumor progression; (2) factors regulated CD38-ecto-enzymatic activity; (3) how CD38positive cells affected the surrounding CD38-negative cells.

It is well known that CD38 is mainly a type II membrane orientation with the catalytic site facing the outside of cells, to consume the $\mathrm{NAD}^{+}$and participant in the regulation of extracellular metabolic molecules, such as adenosine, nicotinamide, and cyclic ADP-ribose (cADPR) [29, 30]. Previous studies have proved that $\mathrm{CD} 38$ via consumed $\mathrm{NAD}^{+}$to generate adenosine as a regulatory hub controls activation, redox homeostasis, pro-inflammatory effects, cell senescence, energy metabolism, and immune suppression of immune cells [31, 32]. Herein we reported that tumor expressing CD38 promoted tumor cell progression via its enzymatic product CADPR independent on $\mathrm{NAD}^{+}$homeostasis and the production of adenosine. Within some specific immune cells, such as neutrophil and DC cells, CADPR was shown to regulate cell migration and trafficking through modulation of calcium influx transit [33]. We identified a role of cADPR to promote cell progression by inducing cytoplasmic calcium flux via TRPM2 channel. Consistent with our study, F. Morandi concluded that inactivation of the CD38-CADPR axis might serve as a novel therapeutic intervention in lung cancer and a recent report indicated that TRPM2 was linked with poor prognosis in patients with pancreatic ductal adenocarcinoma [34, 35]. In addition, to understand if the promotion of CADPR on tumor proliferation, and migration was a generalized phenomenon, we also demonstrated that CADPR could promote cell proliferation and migration in variety of human cell lines which existed the expression of TRPM2. Those observations might suggest that the CD38 enzymatic hydrolysis product CADPR, either from tumor cells or immune cells, might both play an important role for tumor proliferation, formation and migration independent of regulating NAD+ or adenosine in solid TME. It was well known that NRF2 acted as an oncogene, and its principal repressor KEAP1 acted as a tumor suppressor [36-38]. A recent study demonstrated that stimulating KRAS-driven LUAD cancer metastasis was induced by KEAP1 loss but NRF2 activation [24]. In the present study, we also identified CD38-CADPR exhibited a remarkable down-regulation of KEAP1 and upregulation of NRF2 in tumor cells. Furthermore, our results are relevant to the debate on the promises and perils of antioxidant supplementation, especially for lung cancer patients and for the $30 \%$ of them who harbor KEAP1/NRF2 mutations [39]. Therefore, our studies might raise the possibility that targeting CD38 enzymatic activity or downstream CADPR might be effective in patients with NRF2/KEAP1 mutation.
Notably, questions about how to incorporate the enzymatic activity of CD38 into immunotherapeutic strategies still need to be studied in prospective trials. For example, Vaisitti $\mathrm{T}$ elegantly showed that CD38/CD31 interactions could activate cytoplasmic calcium pathway via modulating growth and motility of CLL cells. CD38+ cells pre-incubated with anti-CD31 monoclonal antibody or 8-Br-ADPR could both abrogate CD31 binding to CD38 and block CLL chemotaxis and homing via $\mathrm{Ca}^{2+}$ fluxes mediated by TRPM2 [40]. Since CD38 could consume NAD ${ }^{+}, \mathrm{NAD}^{+}$would be elevated by CD38 knock-out and then might stimulate SIRT1 and PARP1 to induce $T$ cell differentiation into Th1 subsets for enhancing the anti-tumor effects [41]. Moreover, Horenstein A further broadened the horizon for the role $\mathrm{CD} 38$, showing that the enzymatic-product of CD38 was related to the anti-viral and proinflammatory response during the SARS-CoV-2 infection, independent on the CD38/NAD+ axis [42]. Apart from CD38, SARM1 were also confirmed as the additional $\mathrm{NAD}^{+}$-depleting enzyme that using $\mathrm{NAD}^{+}$to produce $\mathrm{CADPR}$, the precise functions of CADPR in those abnormal situations including tumor progression, inflammation, and injury-induced axon death need further characterizations $[43,44]$. In addition, the CADPR might also be produced by CD38 expressed on the surface of immune cells such as tumorassociated macrophages (TAMs) to promote the survival of tumor cells [8].

In conclusion, our findings suggest that selectively targeting the enzymatic activity of CD38 in tumor microenvironment might represent an important strategy in treating non-hematopoietic cancers.

\section{DATA AVAILABILITY}

Specific details will be provided if request.

\section{REFERENCES}

1. Malavasi F, Deaglio S, Funaro A, Ferrero E, Horenstein AL, Ortolan E, et al. Evolution and function of the ADP ribosyl cyclase/CD38 gene family in physiology and pathology. Physiol Rev. 2008;88:841-86.

2. Jiao Y, Yi M, Xu L, Chu Q, Yan Y, Luo S, et al. CD38: targeted therapy in multiple myeloma and therapeutic potential for solid cancers. Expert Opin. Investig. Drugs. 2020;29:1295-308.

3. Raab MS, Engelhardt M, Blank A, Goldschmidt H, Agis H, Blau IW, et al. MOR202, a novel anti-CD38 monoclonal antibody, in patients with relapsed or refractory multiple myeloma: a first-in-human, multicentre, phase 1-2a trial. Lancet Haematol. 2020;7:e381-e394.

4. Vivek Verma V, Shrimali RK, Ahmad S, Dai W, Wang H, Lu S, et al. PD-1 blockade in subprimed CD8 cells induces dysfunctional PD-1+CD38hi cells and anti-PD-1 resistance. Nat Immunol. 2019;20:1231-43.

5. Chen L, Diao L, Yang Y, Yi X, Rodriguez BL, Li Y, et al. CD38-Mediated immunosuppression as a mechanism of tumor cell escape from PD-1/PD-L1 Blockade. Cancer Disco. 2018;8:1156-75.

6. US National Library of Medicine. ClinicalTrials.gov, (2019), https://clinicaltrials.gov/ ct2/show/results/NCT03023423.

7. Hogan KA, Chini CCS, Chini EN. The Multi-faceted Ecto-enzyme CD38: roles in immunomodulation, cancer, aging, and metabolic diseases. Front Immunol. 2019;10:1187.

8. Chini CCS, Peclat TR, Warner GM, Kashyap S, Espindola-Netto JM, de Oliveira GC, et al. CD38 ecto-enzyme in immune cells is induced during aging and regulates NAD + and NMN levels. Nat Metab. 2020;2:1284-304.

9. Deng QW, Zhang J, Li T, He WM, Fang L, Lee HC, et al. The transferrin receptor CD71 regulates type II CD38, revealing tight topological compartmentalization of intracellular cyclic ADP-ribose production. J Biol Chem. 2019;294:15293-303.

10. Lee $\mathrm{HC}$, Zhao YJ. Resolving the topological enigma in $\mathrm{Ca} 2+$ signaling by cyclic ADP-ribose and NAADP. J Biol Chem. 2019;294:19831-43.

11. Dai Z, Zhang XN, Nasertorabi F, Cheng Q, Li J, Katz BB, et al. Synthesis of site-specific antibody-drug conjugates by ADP-ribosyl cyclases. Sci Adv. 2020;6:eaba6752.

12. Munshi $C$, Aarhus R, Graeff R, Walseth TF, Levitt $D$, Lee HC. Identification of the enzymatic active site of CD38 by site-directed mutagenesis. J Biol Chem. 2000;275:21566-71.

13. Fliegert R, Riekehr WM, Guse AH. Does cyclic adp-ribose (cADPR) activate the non-selective cation channel TRPM2? Front Immunol. 2020;11:2018. 
14. Galione A, Chuang KT. Pyridine nucleotide metabolites and calcium release from intracellular stores. Adv Exp Med Biol. 2020;1131:371-94.

15. Tarragó MG, Chini CCS, Kanamori KS, Warner GM, Caride A, de Oliveira GC, et al. A Potent and specific CD38 inhibitor ameliorates age-related metabolic dysfunction by reversing tissue NAD+ decline. Cell Metab. 2018;27:1081-1095.e10.

16. Liu X, Grogan TR, Hieronymus H, Hashimoto T, Mottahedeh J, Cheng D, et al. Low CD38 identifies progenitor-like inflammation-associated luminal cells that can initiate human prostate cancer and predict poor outcome. Cell Rep. 2016;17:2596-606.

17. Bu X, Kato J, Hong JA, Merino MJ, Schrump DS, Lund FE, et al. CD38 knockout suppresses tumorigenesis in mice and clonogenic growth of human lung cancer cells. Carcinogenesis. 2018;39:242-51.

18. Fortunato O, Belisario DC, Compagno M, Giovinazzo F, Bracci C, Pastorino U, et al. CXCR4 Inhibition counteracts immunosuppressive properties of metastatic NSCLC stem cells. Front Immunol. 2020;11:02168.

19. Gross S, Mallu P, Joshi H, Schultz B, Go C, Soboloff J. $\mathrm{Ca}^{2+}$ as a therapeutic target in cancer. Adv Cancer Res. 2020;148:233-317.

20. Yu P, Liu Z, Yu X, Ye P, Liu H, Xue X, et al. Direct gating of the TRPM2 channel by CADPR via specific interactions with the ADPR binding pocket. Cell Rep. 2019;27:3684-3695.e4.

21. Rojo de la Vega M, Chapman E, Zhang DD. NRF2 and the hallmarks of cancer. Cancer Cell. 2018;34:21-43.

22. Lignitto L, LeBoeuf SE, Homer H, Jiang S, Askenazi M, Karakousi TR, et al. Nrf2 Activation promotes lung cancer metastasis by inhibiting the degradation of bach1. Cell. 2019;178:316-329.e18.

23. Shen R, Martin A, Ni A, Hellmann M, Arbour KC, Jordan E, et al. Harnessing clinical sequencing data for survival stratification of patients with metastatic lung adenocarcinomas. JCO Precis Oncol. 2019;18:PO.18.00307.

24. Rodrigo R, Sánchez-Rivera FJ, Westcott PMK, Mercer KL, Bhutkar A, Muir A, et al. KEAP1 mutation renders lung adenocarcinomas dependent on Slc33a1. Nat Cancer. 2020;1:589-602.

25. Bride KL, Vincent TL, Im SY, Aplenc R, Barrett DM, Carroll WL, et al. Preclinical efficacy of daratumumab in T-cell acute lymphoblastic leukemia. Blood. 2018;131:995-9.

26. Lokhorst HM, Plesner T, Laubach JP, Nahi H, Gimsing P, Hansson M, et al. Targeting CD38 with daratumumab monotherapy in multiple myeloma. N Engl J Med. 2015;373:1207-19.

27. Sanchorawala V, Sarosiek S, Schulman A, Mistark M, Migre ME, Cruz R, et al. Safety, tolerability, and response rates of daratumumab in relapsed $\mathrm{AL}$ amyloidosis: results of a phase 2 study. Blood. 2020;135:1541-7.

28. Naeimi Kararoudi M, Nagai Y, Elmas E, de Souza Fernandes Pereira M, Ali SA, Imus $\mathrm{PH}$, et al. CD38 deletion of human primary NK cells eliminates daratumumabinduced fratricide and boosts their effector activity. Blood. 2020;136:2416-27.

29. Horenstein AL, Chillemi A, Quarona V, Zito A, Roato I, Morandi F, et al. $\mathrm{NAD}^{+}$-Metabolizing ectoenzymes in remodeling tumor-host interactions: the human myeloma model. Cells. 2015;4:520-37.

30. Baum N, Fliegert R, Bauche A, Hambach J, Menzel S, Haag F, et al. Daratumumab and nanobody-based heavy chain antibodies inhibit the ADPR cyclase but not the NAD+ hydrolase activity of CD38-expressing multiple myeloma cells. Cancers (Basel). 2020;13:76.

31. Navas LE, Carnero A. NAD+ metabolism, stemness, the immune response, and cancer. Signal Transduct. Target Ther. 2021;6:2.

32. Xie N, Zhang L, Gao W, Huang C, Huber PE, Zhou X, et al. NAD+ metabolism: pathophysiologic mechanisms and therapeutic potential. Signal Transduct. Target Ther. 2020;5:227.

33. Partida-Sanchez S, Gasser A, Fliegert R, Siebrands CC, Dammermann W, Shi G, et al. Chemotaxis of mouse bone marrow neutrophils and dendritic cells is controlled by adp-ribose, the major product generated by the CD38 enzyme reaction. J. Immunol. 2007;179:7827-39.

34. Lin R, Wang Y, Chen Q, Liu Z, Xiao S, Wang B, et al. TRPM2 promotes the proliferation and invasion of pancreatic ductal adenocarcinoma. Mol Med Rep. 2018;17:7537-44.

35. Morandi F, Horenstein AL, Malavasi F. The key role of NAD+ in anti-tumor immune response: an update. Front Immunol. 2021;12:658263.

36. Liu Y, Lang F, Yang C. NRF2 in human neoplasm: cancer biology and potential therapeutic target. Pharm Ther. 2021;217:107664.

37. Ren X, Zou L, Zhang X, Branco V, Wang J, Carvalho C, et al. Redox signaling mediated by thioredoxin and glutathione systems in the central nervous system. Antioxid Redox Signal. 2017;27:989-1010.

38. Davoli T, Xu AW, Mengwasser KE, Sack LM, Yoon JC, Park PJ, et al. Cumulative haploinsufficiency and triplosensitivity drive aneuploidy patterns and shape the cancer genome. Cell. 2013;155:948-62.

39. Berger AH, Brooks AN, Wu X, Shrestha Y, Chouinard C, Piccioni F, et al. Highthroughput phenotyping of lung cancer somatic mutations. Cancer Cell. 2016;30:214-28.
40. Vaisitti T, Audrito V, Serra S, Buonincontri R, Sociali G, Mannino EP, et al. The enzymatic activities of CD38 enhance CLL growth and trafficking: implications for therapeutic targeting. Leukemia. 2015;29:356-68.

41. Chatterjee S, Daenthanasanmak A, Chakraborty P, Wyatt MW, Dhar P, Selvam SP, et al. CD38-NAD+ axis regulates immunotherapeutic anti-tumor $T$ cell response. Cell Metab. 2018;27:85-100.e8.

42. Horenstein A, Faini AC, Malavasi F. CD38 in the age of covid-19: a medical perspective. Physiol Rev. 2021, https://doi.org/10.1152/physrev.00046.2020.

43. Gerasimenko M, Cherepanov SM, Furuhara K, Lopatina O, Salmina AB, Shabalova $A A$, et al. Nicotinamide riboside supplementation corrects deficits in oxytocin, sociability and anxiety of CD157 mutants in a mouse model of autism spectrum disorder. Sci Rep. 2020;10:10035.

44. Jiang $\mathrm{Y}$, Liu $\mathrm{T}$, Lee $\mathrm{CH}$, Chang $\mathrm{Q}$, Yang J, Zhang Z. The NAD+-mediated selfinhibition mechanism of pro-neurodegenerative SARM1. Nature. 2020;588:658-63.

\section{ACKNOWLEDGEMENTS}

We thank Prof. Guideng Li and Prof. Sudan He (Center for Systems Medicine, Institute of Basic Medical Sciences, Chinese Academy of Medical Sciences \& Peking Union Medical College, Beijing 100005, China; Suzhou Institute of Systems Medicine, Suzhou, 215123, China.) for providing plasmids and technical assistance. Dr. Long Gao especially wishes to thank his lovely wife Rui Yin, who always silently be there for him over the past years.

\section{AUTHOR CONTRIBUTIONS}

Long Gao, F. Xiao-Feng Qin, Fang Meng, and Lianjun Zhang conceived the study. Long Gao, Yuan Liu, Xiaohong Du, Sai Ma, Minmin Ge, and Wu Zhao acquired samples and performed the experiments and data analysis. Long Gao, Haijun Tang, Chenfeng Han, Xin Zhao, and Yun Shao analyzed and interpreted the data. Long Gao, F. Xiao-Feng Qin, and Lianjun Zhang wrote and revised the manuscript. All authors read and approved the final manuscript.

\section{FUNDING}

This work was supported by: The National Natural Science Foundation of China (Grant 81701567, 81773058 and 31800726); The Chinese Academy of Medical Sciences Initiative for Innovative Medicine (Grant CAMS-I2M, 2016-I2M-1-005); National grand Foreign Experts projects (G20190001633 and G20190001639); the Project of Sichuan Provincial Education Department (18ZB0174).

\section{ETHICS STATEMENT}

All animal experiments were agreed with animal care, handling, and treatment according to the guidelines approved by the Institutional Animal Care and Use Committee of Suzhou Institute of Systems Medicine (ISM-IACUC-0003-R). The cancerous and non-cancerous pleural effusion were obtained from the patients according to the guidelines approved by the Institutional Care and Use Committee of the First Hospital of Jilin University (2019-025).

\section{CONSENT FOR PUBLICATION}

We confirm that the manuscript has not been published elsewhere and is not under consideration by other journals. All authors have contributed significantly to the work, and have read and agreed on the content of the final version of the manuscript.

\section{COMPETING INTERESTS}

The authors declare no competing interests.

\section{ADDITIONAL INFORMATION}

Supplementary information The online version contains supplementary material available at https://doi.org/10.1038/s41419-021-03968-2.

Correspondence and requests for materials should be addressed to F.M. or F.X-F.Q.

Reprints and permission information is available at http://www.nature.com/ reprints

Publisher's note Springer Nature remains neutral with regard to jurisdictional claims in published maps and institutional affiliations. 
Open Access This article is licensed under a Creative Commons Ac Attribution 4.0 International License, which permits use, sharing,
adaptation, distribution and reproduction in any medium or format, as long as you give appropriate credit to the original author(s) and the source, provide a link to the Creative Commons license, and indicate if changes were made. The images or other third party material in this article are included in the article's Creative Commons license, unless indicated otherwise in a credit line to the material. If material is not included in the article's Creative Commons license and your intended use is not permitted by statutory regulation or exceeds the permitted use, you will need to obtain permission directly from the copyright holder. To view a copy of this license, visit http://creativecommons. org/licenses/by/4.0/.

(c) The Author(s) 2021 